\title{
Temporal Convergence in Shared Networked Narratives: The Case of Blast Theory's Day of the Figurines
}

\author{
Steve Benford and \\ Gabriella Giannachi
}

n this article we introduce a conceptual framework that emerged from our study of an interactive game for mobile participants, called Day of the Figurines, which is played using Short Message Service (SMS). We discuss how this framework may be useful for the management of shared networked narratives over prolonged periods of time. This framework is structured around five "layers of time," ranging from the temporal organization of a story universe as conceived by its author(s) to the way in which this universe is ultimately perceived or even reconstructed by a participant, possibly as a result of experiencing multiple interleaved narrations of the story in different forms and at different times. Our framework, which will be useful for reasoning about the temporal structures of interactive media works, was initially conceived to assist the analysis and understanding of the operation of time in such experiences, although we envisage that in the long term it might potentially also be used as part of interactive media design.

\section{DAY OF THE FIGURINES}

Day of the Figurines (2006-) is a narrative-driven text messaging game for mobile phones created by Blast Theory, a U.K.-based company led by Matt Adams, Ju Row Farr and Nick Tandavanitj that, since the late 1990s, has developed influential mixedmedia performances, games and installations [1] in collaboration with the University of Nottingham's Mixed Reality Laboratory, Sony and the Fraunhofer Institute as part of the European Union's Integrated Project on Pervasive Games (IPerG), that is, games that interweave digital media with everyday life experiences [2]. The game represents the primary, if not the only, example of these technologies being deployed in a pervasive artwork for mobile audiences run entirely through SMS. Previous works by Blast Theory, all developed in collaboration with the Mixed Reality Laboratory, explored the themes of spatial expansion. Thus Can You See Me Now? (2003-2005), winner of the Golden Nica (2003) at Ars Electronica, utilized the Global Positioning System (GPS) to track performers' movements through a city and then map these onto the movements of their avatars through a corresponding digital model of this city as part of a game of chase with on-line participants.

Steve Benford (computer scientist), Mixed Reality Laboratory, University of Nottingham, Nottingham, NG81BB, U.K. E-mail: <sdb@cs.nott.ac.uk>.

Gabriella Giannachi (performance studies and new media theorist), Centre for Intermedia, University of Exeter, Exeter, EX44LA, U.K. E-mail:<G.Giannachi@exeter.ac.uk>.
Other works have explored social expansions, including Uncle Roy All around You (2003), which engaged with the boundaries between the fictional world of a game and the physical reality of a city, implicating bystanders on the city streets in its narratives. The developers of Day of the Figurines set out to explore the theme of the temporal expansion of pervasive games.

To participate in the game, players visit a physical space, where they find a small square white table with 1,000 neatly arranged figurines and, close to it, a large-scale white metal model of an imaginary town with 50 destinations (Fig. 1). The figurines, the size of a thumbprint, are cartoon-like, while the destinations, which are cut out of the surface and folded vertically to form white silhouettes, are based on a typical British town and include, for instance, a 24 Hour Garage, a Boarded up Shop, a Hospital and Kath's Café (Fig. 2). Two video projectors beneath the surface of the board shine through holes in the table and reflect off mirrors mounted horizontally above it, enabling the surface of the table to be augmented with projections of live information from the game (Color Plate B No. 3). While registering for the game, each participant selects a figu-

Fig. 1. Blast Theory, Day of the Figurines, 2006-. (@ Blast Theory. Photo (c) University of Nottingham.) A player choosing a figurine at Sonar, Barcelona.

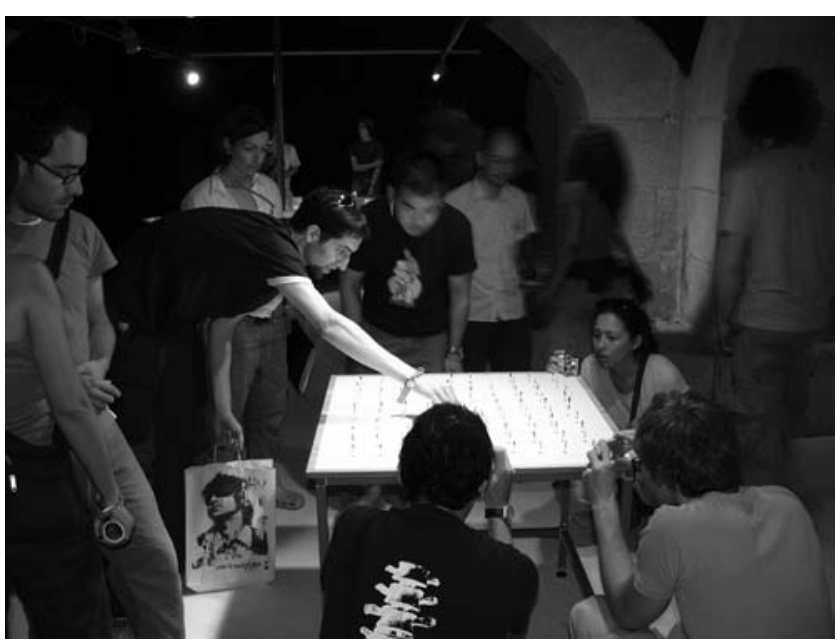




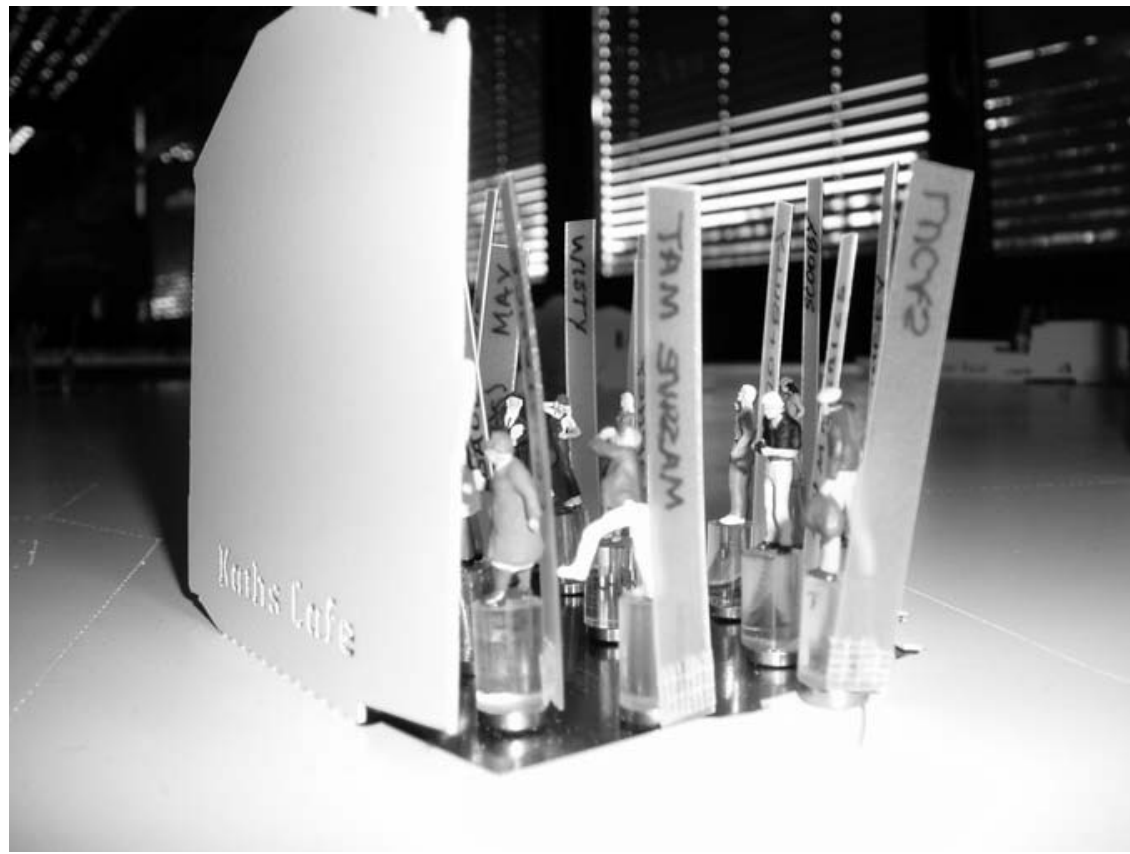

Fig. 2. Blast Theory, Day of the Figurines, 2006-. Game destination Kath's Café at Hebbel am Ufer, Berlin. (@ Blast Theory. Photo @) Gabriella Giannachi.)

rine and creates a role for it (see Fig. 1). Participants then send and receive SMS messages to control their figurines as they live through a "day" in the life of the fictional town, visiting destinations, observing events, using objects, responding to dilemmas, undertaking missions and talking with others (Fig. 3). The game balances a pre-scripted narrative with interactivity, and the experience is fundamentally narrative driven, following a pre-scripted storyline. Participants are refugees who are dropped off in the town in the early morning. As the day unfolds, they experience a sequence of scheduled events, including a fete, the discovery of two dead lovers at the cemetery, a riotous gig and an army sweeping into town. These scripted events are interspersed with interactive elements of gameplay, such as multiple-choice dilemmas and missions that require participants to visit destinations, find and use objects, communicate with each other and maintain their health. Each element has a temporal scope that constrains when in game time it becomes available to participants. It may also have a timeout, after which a default answer or action is assumed so the narrative can move on. Missions and dilemmas need to be resolved within given set times, while the overall pace of the game is structured around the passing of a day. Finally, Day of the Figurines does not entail a conventional dramatic or game conflict, nor does it result in a clearly quantifiable outcome. Rather, the piece follows less formal play activities, and participants spend most game time advising each other on what to do in the game (see Fig. 3).

From its conception, Day of the Figurines adopted a distinctive approach to the treatment of time. The experience was designed to unfold in the background of participants' daily lives, reflecting the way in which mobile phones enable us to finely interweave different threads of activity. It was also designed to accommodate the distinctive nature of SMS, which typically involves asynchronous, intermittent, short, costly and infrequent messages. The artists therefore aggregated several aspects of the pre-scripted narrative, such as a resume of the participants' status and an update of game events, into single SMS, and accommodated dramaturgically the fact that participants, who were mobile and often texting while on the go, would send and receive only a few messages each day as part of their pervasive experience. The game deliberately slowed down fictional time so that the 24 hours of time in the narrative were played out over 24 days of its participants' lives. The team intended for all participants to share the same objective game time, which unfolds linearly in relation to actual time, no matter how much or little each individual played. Indeed, a participant's figurine remained active in the game even when the participant's phone was switched off or lost its network connection, with participants receiving SMS notifications of any missed events the next time they reconnected to the game. Finally, the game was delivered as an event-based touring performance, be- ing booked to run at hosting venues for fixed periods of time. Each performance ran for 24 days, between set start and end dates, for 10 hours a day (while the venue was open), with interactive play being suspended outside of these times.

To date, Day of the Figurines, which received an honorary mention at Prix Ars Electronica 2007, has been performed in Berlin, in Singapore and three times in the U.K. for over 1,000 participants altogether. The experience revealed some interesting issues with regard to the piece's temporal structure. First, the majority of participants interacted episodically, dipping in and out of the game, sometimes not actively engaging for several days before becoming active again. Patterns of engagement also varied greatly among participants due to variations in their preferences for where and when to take part, different kinds of phone use and shifting personal circumstances over the course of a month. Messages could be delayed for hours before being delivered to participants due to network congestion, lack of coverage or phones being switched off. Some participants were irritated by a sudden flood of messages as they switched their phones back on after a long break, while others reported frustration with playing across time zones, as this led to a mismatch between scheduled opening hours and their own waking hours. This combination of delays, episodic engagement and multiple time zones made it difficult for participants to engage in conversation and maintain social relationships with other players. An orchestration interface developed to manage the game enabled the game operators to control the rate of progression of time in the fictional narrative relative to time in the real world. This feature was used once in Singapore, when the hosting venue was closed for a day; the operators sped up game time over the following few days until the missing fictional hour had been recovered. Finally, participants enjoyed reviewing their histories of interaction by scrolling through saved messages on their own phones or, at the end of the game, revisiting their game histories on the web.

\section{LAYERING TIME}

The use of computers to mediate and deliver interactive artworks such as Day of the Figurines raises significant challenges for the treatment of time. This, as has already been identified by Roy Ascott with respect to electronic spaces created by telecommunications and computer systems, is because in these 
Sent 17:34

GMT Sun 08 Say to dwight what is going on here? Apr

Received

17:39 10:39am, GEOFFREY said: "well i know its early, but $\mathrm{i}$ was just about GMT Sun 082 open my bottle of wine. Anyone fancy a glass?" Apr

Sent 11:19 Pick up sleeping bag. Say to chaos does anyone know how to help the GMT Tue 10 people stuck in the boarded up shop.

\begin{tabular}{|c|c|}
\hline $\begin{array}{r}\text { Sent 18:44 } \\
\text { GMT Thu } 26 \\
\text { Apr }\end{array}$ & $\begin{array}{l}\text { Say anyone around knows what going on? Am i supposed to be helping } \\
\text { the soldiers? Anyone there? }\end{array}$ \\
\hline $\begin{array}{r}\text { Received } \\
\mathbf{1 1 : 1 4} \\
\text { GMT Fri } 27 \\
\text { Apr }\end{array}$ & $\begin{array}{l}\text { 05:00am, the rumour is getting stronger: get to the Rec by } 5.45 \mathrm{am} \text { to } \\
\text { catch a ride out of this town before it's too late. You are feeling very ill. }\end{array}$ \\
\hline $\begin{array}{r}\text { Received } \\
\mathbf{1 2 : 2 0} \\
\text { GMT Fri } 27 \\
\text { Apr }\end{array}$ & $\begin{array}{l}\text { 05:06am, LITTLE ANGEL said: "RALPH are u ok? We should try } 2 \\
\text { go } 2 \text { the rec b4 its } 2 \text { late!" }\end{array}$ \\
\hline
\end{tabular}

Fig. 3. Examples from Jack's game, Lighthouse, Brighton. (@ University of Nottingham)

cases meaning is generated not only by the "send-receive" mechanism but also "out of the negotiations between participants in the system" who can access "this new information space asynchronically - that is without constraints of time or space" [3]. Whereas other telematic artworks adopted computer-mediated telecommunication networks and utilized asynchronous communication, Day of the Figurines did so while also using a semi-structured narrative that, because of the medium broadcasting it, could be experienced by participants on the go. Day of the Figurines shows that dramaturgies defining the temporal setting of a story and the timing of its narration can be broadened to include the times at which participants desire or are available to interact. These may vary greatly when participants are engaged across international networks or through mobile technologies [4]. The ability to record participants' interactions within a given performance and then reuse these interactions in subsequent events also opens up new possibilities for narrative structures that combine present and past occurrences. In order to help us understand the complex interactions between the designed temporal structure of Day of the Figurines and participants' subsequent experience of this structure, we now introduce a conceptual framework to represent the temporal construction of mediated interactive narratives (Fig. 4). Our framework comprises five layers, each of which addresses a different as- pect of temporal structure, ranging from the mapping of time in the fictional universe created by the author to the way in which time is perceived by participants. In between lie three intermediate layers that address the temporal aspects of narration, scheduling and interaction.

The first layer is constituted by story time, which is usually determined by the author and describes the time span defining the narrative outline of the story, organized in chronological order [5]. Games involve their own distinct narrative subdivision, including the distinction between "result time" (games played until someone wins) versus "set time" (games played for a fixed period of time when a winner is declared) [6]. For example, Day of the Figurines' story time encapsulates the life of a contemporary town over a period of 24 fictional hours and operates according to an overall set time, although some of its missions and dilemmas, and the narratives they summarize, are structured around result time.

The second layer consists of plot time, which describes the temporal structure of the narration of the story, that is, the timing and ordering of events in their re-presentation. This need not be the same as the timing and order of the story. For example, dramatic texts may adopt "open" structures, compressing story time into a shorter plot time, which then refers to events outside of plot time that influence the course of the plot's events, while other texts assume "closed" structures in which the story is self-contained, with no background events influencing the beginning, and the ending being final. Plots may contain subplots defined through different temporal narrative orders, which may vary according to given set and result times. Film and television narrative structures also compress time ("ellipsis") and alter the ordering of events as they are narrated (e.g. "flashbacks"). In turn, interactive media have introduced their own distinctive structures in the form of multi-threaded hypertext plots and the looping structures of computer games, which may be combined with more traditional filmic elements. Adopting film terminology [7], the structure of plot time is determined by the director (who may be the same person as the author or even the performer). The more interactive the work, the more the participant will be able to author their own plot, determining its temporal construction and influencing its perception by other players. In Day of the Figurines, unconventionally, plot time expands story time, mapping 24 hours of the former onto 24 days of the latter. This allows participants to author their own plots and subplots within the overall narrative framework according to a number of variable factors, such as how much free time they have in their everyday lives and what kind of pace (e.g. setor result time-based) they may wish to experience.

The third layer consists of schedule time, which is controlled by the producer, curator or publisher and describes the times at which the narration is made available to participants, be they readers, viewers or players. All media involve a schedule time (plays and films are shown at set times), but it is in television that schedule time, through its relationship to channels and advertising sponsorship, takes on a particular significance, to the point where it strongly influences the form of the underlying story and plot, as seen by the rise of TV series and serials. As an example, Day of the Figurines is scheduled to be performed for 10 hours a day beginning on set "opening days," although this can vary due to local circumstances such as a venue being closed.

The fourth layer consists of interaction time, which describes the times at which participants engage with the story once it has been made available, either as a matter of choice (playing at times that suit them) or as a result of technical constraints (such as network availability and delay). Interaction time is primarily controlled by the participant. Research in the field of human-computer interaction (HCI) has produced various ac- 
counts of interaction time in narrative experiences, including studies of how players' engagement with long-term mobile games adapts to the ongoing patterns of their daily lives-for example, their commuting [8]. Interaction time in Day of the Figurines is characterized by largely episodic patterns of play, with players frequently disengaging and reengaging over a prolonged period of time.

Finally, perceived time, building on Alan Dix's work on user perception and action within an HCI context [9], refers to the way in which the timing of events may be perceived by individual participants. For this concept, it is useful to refer to Francisco Varela's study of the specious present. Inspired by Edmund Husserl, Varela talks about perceived time as having a three-part structure, based on now, retention and protention. Retention is described as belonging to the past even though it is happening now, while protention is portrayed as "the expectation or the construction of the future" [10]. Studies of computer gaming have noted that players may enter a "flow state," in which their perception of clock time appears to be altered [11]. Whereas "flow" indicates that the present moment can be perceived as a duration, though not necessarily one that corresponds to clock time, the "now, retention and protention" structure points to the possibility that the experience of perceived time could be based on tenses or trajectories.

Analyzing player behavior in Day of the Figurines, it is notable that participants engaged with the game episodically (Fig. 4), weaving in and out of various embedded and emergent subplots, thus playing within pre-authored set times but also authoring their own set- or result time-based micro-temporalities, while at the same time maintaining an eye on the game's overall story time by interacting within the given scheduled times [12]. While this suggests that participants were able to play within a multiplicity of times, selecting or being moved between given temporal trajectories, little is still known about how they themselves perceived these trajectories to work within the fabric of their everyday lives and whether indeed this temporal expansion had any effect on their perception of clock time both within and outside the world of the game.

\section{ANALYZING THE TEMPORAL FRAMEWORK}

We now offer three general observation about this layered framework. First, we propose that a holistic analysis or design of time in an interactive narrative experience requires recognizing or constructing appropriate structures for plot, schedule and interaction time and the ways in which they interact with one another so as to establish a particular mapping between story time and perceived time. For instance, the distinctive temporal qualities of Day of the Figurines emerge from the way in which story time is mapped onto plot time and is further shaped by a schedule of performances, which are in turn subject to the temporal patterns of episodic play and finally experienced by participants. In other words, a powerful interactive narrative experience requires an understanding of how story time can be related through plot time, which in turn is affected by a schedule and then by patterns of interaction and finally by the ways in which participants perceive time.

Second, we suggest that there is longer-

Fig. 4. The five layers of time in interactive narrative. (@ Steve Benford)

\section{Time as perceived by the participant}

$\Uparrow \begin{aligned} & \begin{array}{l}\text { Perceived time } \\ \text { (participant) }\end{array} \\ & \begin{array}{l}\text { Interaction time } \\ \text { (participant) }\end{array} \\ & \text { Schedule time } \\ & \text { (scheduler) } \\ & \text { Plot time } \\ & \text { (director) } \\ & \text { Story time } \\ & \text { (author) }\end{aligned}$

The timing of interactions as perceived
by the participant

The times at which a participant chooses or is able to interact

The times at which the narration is made available

The temporal structure of the narration of the story

The temporal structure of the underlying story world
Time as conceived by the author term "back-pressure" between these five layers in the sense that our emerging understandings of higher layers ultimately shape the nature of the lower ones. As a concrete example, television theorists [13] attribute the rise of new story and plot forms such as series and serials to the impact of TV schedules, showing how the nature of schedule time has exerted a back-pressure on the structure of plot time. Looking to the future, we predict that a growing understanding of interaction and perceived time will ultimately lead to new structures for schedule and plot time that are adapted to the demands of highly episodic play. Indeed, the design of Day of the Figurines already shows some evidence of this in the way that it deliberately accommodated episodic play through small, bite-sized scripted events and dilemmas that were responsively allocated to players whenever they chose to engage.

Finally, we can also relate this framework to notions of the divergence and (re)convergence of media over longer timeframes. It is now commonplace for a story to be retold through different media forms that are often interleaved, remediating earlier narratives that possibly existed in some other form or medium. While medium-specific dramatic structures are unable to address these complex layers of intertextual reference, our hybrid framework is able to map multiple temporal structures flexibly onto one another.

\section{TOWARD A PluRality OF TIMES}

In the aftermath of the temporal revolution brought on by industrialization [14] and later of the theorization of quantum theory and relativity [15], time became once again the subject of renowned philosophical and social-science investigations, particularly in the 1960s and then again from the 1990s on. These investigations have identified at least two distinct phases, marking the invention of new technologies of time and, concurrently, the appearance of new social and economic groups. Thus, Jacques Le Goff identified how a time of the Church was displaced in medieval cities by "a time of merchants" [16]. Similarly, Claude LéviStrauss distinguished between times of societies adopting non-cumulative, simultaneous, cyclical temporal structures and a Western time dominated by history [17], while Maurice Bloch differentiated between "a universal linear time" and a "relative cyclical time," "one associated 
with everyday practice, the other with ritual and ideology" [18]. While agricultural societies are inclined to adopt cyclical conceptions of time, so-called recordkeeping practices cause a society to tend toward cumulative or linear ones [19]. E.P. Thompson further distinguished between task-structured time characteristic of preindustrial societies and industrial time, defined by linear time [20]. A primary goal during the industrial period was to gain control over production time; thus a universal unit of time was deemed necessary to distinguish work time from free time [21]. This separation between work and free time is distinctive of this phase of Western economic production and is reflected in the way that most forms of entertainment were associated with free time and separated out from the flow of everyday life through their occurrence within dedicated buildings. Such forms also necessitated fixed temporal dramaturgies reflecting a singlepoint perspective, which marked the difference between the world of fiction and that of everyday life.

While some scholars have acknowledged a chronology of time and have shown how different practices of time have shaped the organization of all layers of society, from work to entertainment and religion to ideology, others have identified the possibility that these different types of time may have been operating successively but also, to some extent, in synchronicity. Among these are Barbara Adam, who notes that temporal constructions "cannot be contained within the classical dualisms of social science analysis" and that in fact a "multitude of times coexist" [22], and Jacques Attali, who claims that "one time structure never eliminated another; each new structure was overlaid on top of the previously dominant structure" [23]. A number of scholars have argued that novel, flexible, subject-oriented temporal structures are appearing in postindustrial societies. Olivier Klein, for instance, notes that a new time structure has started to exist distinctively "alongside industrial time" and shows that marked differences exist between this new type of time and industrial time. Among them, he cites a blurring between work time and social time, which, as we had seen, was a feature of the industrial period, and the subsequent increase in the interaction between work and personal activities, including a return to task-oriented work [24]. Likewise, Attali proposes the advent of a new "time of codes," which, he claims, will be superseding the time of machines. Interestingly, he refers to the possibility of a "time specific to each system" and suggests that

it is possible to see how the dual nature of the emerging time structure could constitute one of the primary foundations of the autonomy/integration organizational model by combining within each unit of the production system an "autonomous" time, specific to each component, and a collective time with an integrating role in the structure as a whole [25].

Three fundamental concerns emerge from these analyses, all of which informed the thinking at the heart of this study of Day of the Figurines: First, these new frameworks coexist alongside linear industrial models dependent on a "universal" clock time and relative cyclical times; second, there is no longer a separation between work time and social time, and in fact time can be system specific; third, despite this specificity, a convergence between these semiautonomous systems is necessary, which means that some kind of integration between fairly independent subject-specific temporal structures and an overarching collective framework needs to occur.

\section{CONCLUSION}

The leading theorist of information society, Manuel Castells, observes the formation of two new forms of space and time, which he calls "space of flows," allowing for simultaneous social interaction at a distance, and a "timeless time," referring to "the desequencing of social action, either by the compression of time or by the random ordering of the moments of the sequence." This, he argues, could lead to the possibility of saturating time with social practice "by inserting communication into all the moments when other practices cannot be conducted, such as 'in-between' time during transportation, in waiting line, or simply during free time" so that individuals would have to organize their activities according to "flexible compartments of time" [26]. Robert Hassan and Ronald Purser have likened these spaces or "flows" producing "timeless time" to the temporal ecology of networks and claimed that people are able to communicate in "real time," thus creating "a temporal context where what the local time of the clock reads is of no importance" [27]. Following on from these investigations, it becomes evident that not only should there coexist semiautonomous temporal systems, within which a multiplicity of times cohabit and operate in conjunction to an overarching collective framework, but also these sys- tems should be able to allow the temporally and spatially dispersed community that utilizes them to operate episodically and semi-independently within its own space/times while maintaining a manageable relationship to clock time. In other words, it appears that postindustrial time, Attali's time of codes, is not only a tool for social interaction but also, as in the case of Day of the Figurines, a means toward the experience of different types of time through an intermedial convergence in which the inter-relationship of entertainment, work and everyday life is orchestrated globally, respecting the flexibility, autonomy and creativity of dispersed and mobile subjects. In this context, time not only shapes participants' experience of the artwork relative to the subject who is interacting with the work but in fact becomes part of its content and form. A framework synchronizing the trajectories by which authorial or embedded times (but also plot-based embedded or emergent temporal structures) are set against scheduled yet possibly divergent clock times will therefore prove useful for the management of mobile forms of entertainment in the age of media convergence. Further research into its effects on participants' desire or ability to interact with one another, and their perception of such a complex temporal universe, should prove an exciting new field of investigation.

\section{Acknowledgments}

We gratefully acknowledge the support of the IPerG project $<$ www pervasive-gaming.org $>$, funded under project <www.pervasive-gaming.org $>$, funded under the European Union's Framework VI Information
Society Technologies program. Research for this article was also conducted in the context of "Performing Presence: From the live to the simulated, a 4-year project funded by the Arts and Humanities Research Council, U.K < presence stanford edu $>$. We are indebted to Blast Theory for generous feedback are indebed to Bast Theory for generous feedback and to Nick Kaye and Peter Thomson for alerting us to subject-specific literature.

\section{References}

Unedited references as provided by the authors.

1. Blast Theory: <www.blasttheory.co.uk/>

2. M. Capra et al., "The Multimedia Challenges Raised by Pervasive Games," MM'05, 6-11 November 2005, Singapore, 89-95.

3. R. Ascott, Electra: Electricity and Electronics in the Art of the Twentieth Century (Paris, Musée d'Art Moderne de la ville de Paris: 1983), p. 398.

4. See also J. Murray, "From Game-Story to Cyberdrama," in N. Wardrip-Fruin and P. Harrigan, Firs Person (Cambridge, MA, MIT Press: 2004), pp. 2-12.

5. M. Pfister, The Theory and Analysis of Drama, tr. J Halliday (Cambridge: CUP: 1998).

6. P. Thomson, "Games and Plays-An Approach to Ionesco," Educational Theatre Journal, 22:1, 1970 , to Ionesco. 
7. R.C. Allen, Channels of Discourse, Reassembled (London and New York, Routledge: 1992).

8. M. Bell, M. Chalmers, et al., "Interweaving Mobile Games with Everyday Life," CHI 2006, Montréal, Canada, ACM, 2006, 417-426.

9. A. Dix, "The Myth of the Infinitely Fast Machine," in D. Diaper and R. Winder (eds), People and Comput ers III (Cambridge, CUP: 1987), pp. 215-228.

10. F.J. Varela, "The Specious Present: A Neurophenomenology of Time Consciousness," in J. Petiot et al. (eds), Naturalizing Phenomenology (Stanford, Stanford University Press: 1999), p. 9 and p. 12.

11. J. Chen, "Flow in Games (and everything else)," Communications of the ACM, 50:4, April 2007, 31-34.

12. S. Benford and G. Giannachi, "Temporal Trajectories in Shared Interactive Narratives," Proceeding ACM SIGCHI Conference on Human Factors in Comput ing Systems (CHI 2008), ACM Press, <www.acm.org>.

13. Allen [7].

14. S. Kern, The Culture of Time and Space: 1880-1918 Cambridge Massachusetts: Harvard University Press, 1983)

15. W. Heisenberg, Physics and Philosophy (London, Penguin: 1990 [1962]).

16. J. Le Goff, “Au Moyen-Âge: Temps de l'Eglise et temps du marchand," Annals E.S.C. XV, 1960 417-433.
17. C. Lévi-Strauss, Structural Anthropology, tr. C. Jacobson and B. Grundfest Schoepf (Harmondsworth, Penguin: 1972; [1963]), p. 286.

18. M. Bloch, "The Past and The Present in The Present," Man (NS) 13, 1977, 591-599.

19. G.N. Bailey, "Concepts of Time in Quaternar Prehistory," Annual Review of Anthropology, 12, 1983, 165-192.

20. E.P. Thompson, "Time, Work-Discipline and Industrial Capitalism," Past and Present, 38, 1967 56-95.

1. O. Klein, "Social Perception of Time, Distance and High-Speed Transportation," Time and Society, 13:2-3, 2004, 252.

22. B. Adam, Timewatch (Cambridge, Polity Press: 1995), p. 42

23. J. Attali, Histoire du temps (Paris, Fayard: 1982) pp. 247-248.

24. Klein [21] pp. 254-255.

25. Attali [23] pp. 260 and 256.

26. M. Castells, M. Fernández-Ardèvol, et al., Mobile Communication and Societ)' (Cambridge, Massachusetts, MIT Press: 2007), p. 171, p. 174 and p. 176
27. R. Hassan and R. Purser, 24 | 7 Time and Temporal ity in the Network Society (Stanford, Stanford University Press: 2007), p. 10

Manuscript received 5 April 2008.

Steve Benford is Professor of Collaborative Computing at the Mixed Reality Laboratory, Department of Computer Science, University of Nottingham. His research concerns new technologies to support social interaction across computer networks, including artistic and entertainment applications of mobile and ubiquitous computing. He has published over 200 works including 20 papers in the ACM's CHI conference. He won the Golden Nica at Prix Ars Electronica 2003 for his collaboration with Blast Theory on Can You See Me Now?

Gabriella Giannachi is Associate Professor in Performance and New Media at Exeter University. She is author of Virtual Theatres (2004); The Politics of New Media Theatre (2007); and co-author with Nick Kaye of the forthcoming Performing Presence: Between the Live and the Simulated.

\section{CALL FOR PAPERS}

\section{ArtScience: The Essential Connection}

What is the value of artistic practices, techniques, inventions, aesthetics and knowledge for the working scientist? What is the value of scientific practices, techniques, inventions, aesthetics and knowledge for the artist? When does art become science and science, art? Or are these categories useless at their boundaries and intersections?

Can an individual excel at both science and art, or is even a passing familiarity with one sufficient to influence the other significantly? Do the arts ever contribute significantly to scientific progress? Where will current scientific innovations lead the arts in the next few decades?

Submissions exploring these questions can be from artistic scientists who find their art avocation valuable; from scientist-artist collaborators who can demonstrate a scientific or artistic innovation; from scientifically literate artists who draw problems, materials, techniques or processes from the sciences; or from historians of art or science looking at past examples of such interactions.

Interested authors are invited to send proposals, queries and/or manuscripts to the Leonardo editorial office: Leonardo, 211 Sutter St., Suite 501, San Francisco, CA 94108, U.S.A. E-mail: <isast@leonardo.info>. 